\title{
Relationship of Self-Esteem with Quality of Life and Narcissism in Veterans and Disabled Professional Cyclists
}

\section{ART I C LE INF O}

\section{Article Type}

Descriptive Study

\section{Authors}

Zar A. ${ }^{1} P h D$,

Ahmadi M.A.* $M S c$,

Ahmadi $\mathrm{F}^{2} M S C$,

Ataee Gharache N. ${ }^{3} M S c$

How to cite this article
Zar A, Ahmadi M A, Ahmadi F, At-
aee Gharache N. Relationship of
Self-Esteem with Quality of Life
and Narcissism in Veterans and
DisabledProfessionalCyclists. Ira-
nian Journal of War \& Public
Health. 2017;9(2):91-95.

\section{A B S T RA C T}

Aims High self-esteem positively correlated with high quality of life and narcissism. The aim of the study was to investigate the self-esteem and its correlation with quality of life and narcissism in the veteran and disabled professional cyclists participated in the state championship. Instrument \& Methods In the descriptive study, 27 male physically disabled and veteran cyclists, who took part in the state championship in 2016, were studied. The subjects were selected via available sampling method. Data was collected by Rosenberg self-esteem scale, health-related quality of life scale, and Ames narcissistic personality questionnaire. Data was analyzed by SPSS 17 software using Pearson correlation coefficient.

Findings The mean score of self-esteem, mean total score of quality of life, and mean score of narcissism were $6.83 \pm 3.23,73.45 \pm 5.60$, and $5.11 \pm 3.63$, respectively. Self-esteem did not significantly correlate with total score of quality of life and narcissism $(p>0.05)$. However, selfesteem positively and significantly correlated with the vital energy and freshness subscale of the quality of life ( $r=0.481 ; \mathrm{p}=0.017)$.

Conclusion The veteran and disabled professional cyclists participated in the state championship are with acceptable self-esteem and quality of life. Nevertheless, their self-esteem does not correlate with quality of life and narcissism.

Keywords Veterans; Disabled Persons; Self Esteem; Quality of Life; Narcissism
*Exercise Physiology Department, Physical Education and Sport Science Faculty, Shahid Chamran University of Ahvaz, Ahvaz, Iran

${ }^{1}$ Sport Sciences Department, Literature \& Humanities Faculty, Jahrom University, Jahrom, Iran

2Exercise Physiology Department, Physical Education and Sport Science Faculty, Shahid Chamran University of Ahvaz, Ahvaz, Iran ${ }^{3}$ Sport Science Department, Education \& Psychology Faculty, University of Shiraz, Shiraz, Iran

\section{Correspondence}

Address: Physical Education and Sport Science Faculty, Shahid Chamran University of Ahvaz, Golestan Boulevard, Ahvaz. Postal Code: 61357-83151

Phone: +98 (61) 33369512

Fax: +98 (61) 33336316

ahmadi_ma@yahoo.com

\section{Article History}

Received: November 28, 2016

Accepted: January 18, 2017

ePublished: April 24, 2017

\section{I T A T I O N L I N KS}

[1] Relationship among depression, anxiety, stress and personality [2] Relationship between Spiritual health With quality of life veterans [3] Self-concept of Finnish children on the second grade of normal ... [4] Selfesteem, narcissism, and stressful ... [5] The relationship between quality of life ... [6] Relationship between self-esteem ... [7] The relationship between quality [8] Correlation between academic achievement... [9] Comparative study of the relationship between ... [10] Association between physical activity ... [11] Exercise dose-response effects on quality of life ... [12] Comparative of Mental Health Disabled ... [13] Evaluation of sport injury incidence ... [14] Quality of life in Iranian veterans with ... [15] Effect of group exercise program on quality... [16] Changes in the quality of life of veterans ... [17] The effect of regular exercise on self-esteem ... [18] Comparing the effectiveness of rehabilitation ... [19] Validation of the narcissistic personality inventory ... [20] Handbook of sport ... [21] Neuro linguistic programming for ... [22] The relationship of narcissism, self esteem and aggression among adolescence girls and their comparisons in early ... [23] The Relationship between self-esteem and happiness ... [24] A 12-Item shortform health survey ... [25] The Iranian version of 12-item Short Form Health Survey (SF-12): Factor structure, internal consistency and construct ... [26] The NPI-16 as a short measure of ... [27] Iranian validation of the narcissistic personality ... [28] Self-esteem and women with ... [29] The effect of physical activity in the water ... [30] Comparison of the quality of life of paraplegic veterans and disables; case study of mazandaran ... [31] Quality of life among disabled and healthy individuals a comparative ... [32] Effect of playing football (soccer) on balance, strength, and quality of life in unilateral below-knee ... [33] Comparing the quality of life of the disabled participat ... [34] Effectiveness of physical activity on quality of life and pain self-efficacy in veterans and non-veterans with amputations of ... [35] The relation of narcissism and self-esteem ... [36] Self-functioning and perceived parenting: Relations of parental empathy and love inconsistency with narcissism, depression ... 
كاهش سطح عزت نفس هستند كه اين مساله مىتواند باعث إنثا

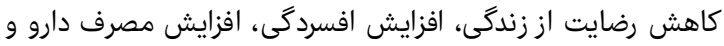

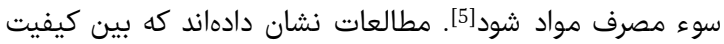

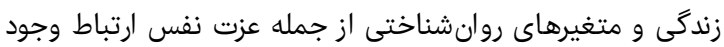

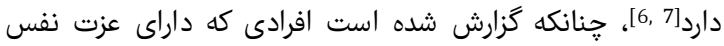

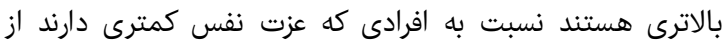

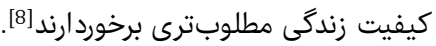

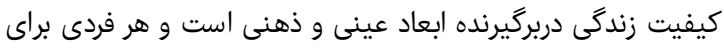

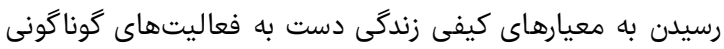

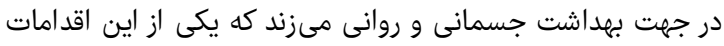

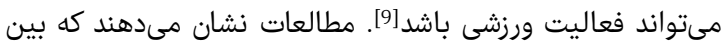

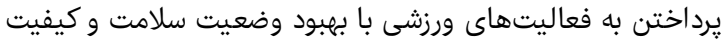

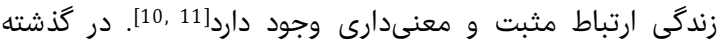

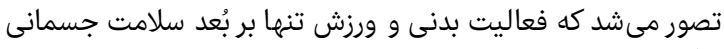

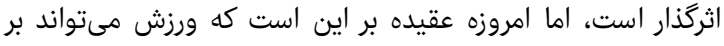

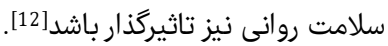

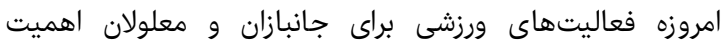

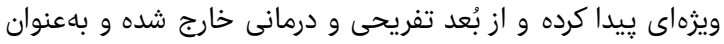

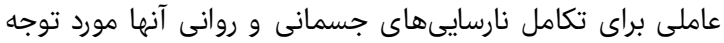

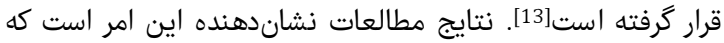
ورزش و فعاليت بدنى نقش بسيار مهمى در سلامت است عمومى مردم

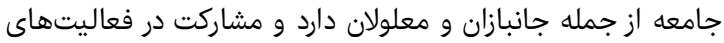

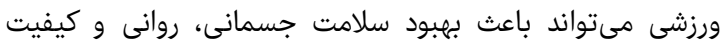

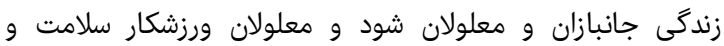

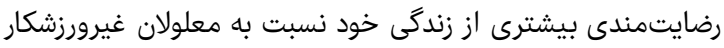

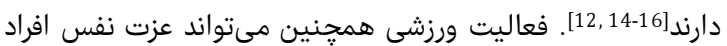

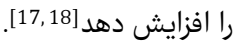

خودشيفتگى يكى از مفاهيم قديمى در درائى روانشناسى و بهويزه

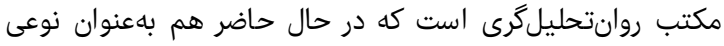

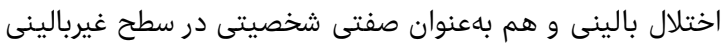

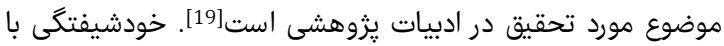

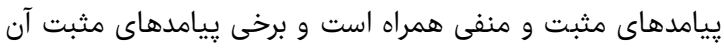

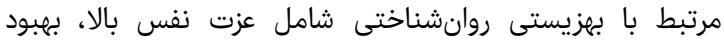

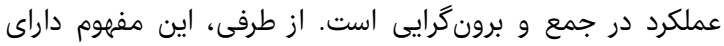

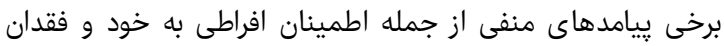

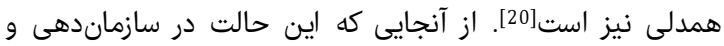

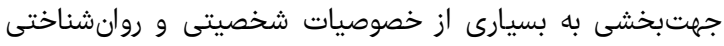

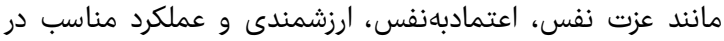

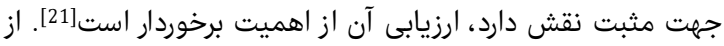

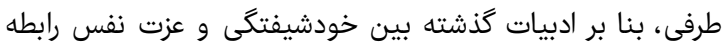

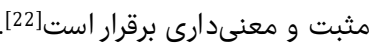

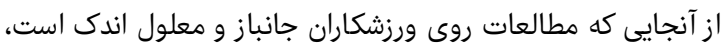

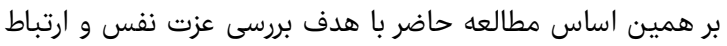

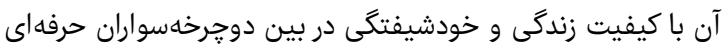

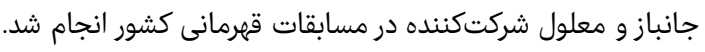

ابزار و روش ها

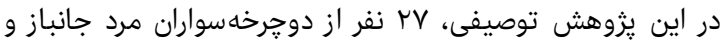

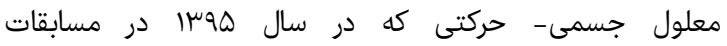

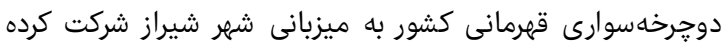

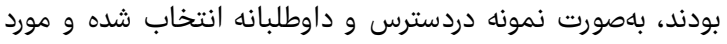

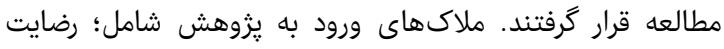

دوره 9، شماره r، بهار عوسا
ارتباط عزت نفس با كيفيت زندكى و خودشيفتگى در دوجرخهسواران حرفهاى جانباز و معلول

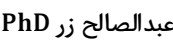
كروه علوم ورزشى، دانشكده ادبيات و علوم انسانى، دانشكاه جهرم، جهرم ، ايران

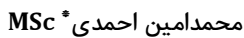
كروه فيزيولوزى ورزش، دانشئ دانشده تربيت بدنى و علوم ورزشى، دانشكاه شهيد تمران اهواز، اهواز، ايران

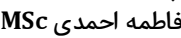

كروه فيزيولوزى ورزش، دانشكان إنداه تربيت بدنى و علوم ورزشى، دانشكاه شهيد

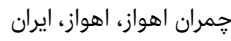

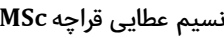
كروه علوم ورزشى، دانشكده علوم تربيتى و روان شناسى، دانشكاه شيراز، شيراز،

جكيده

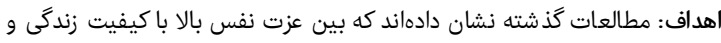

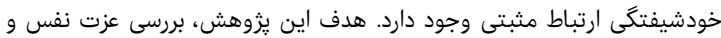

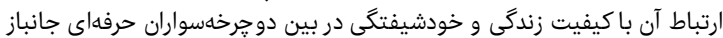

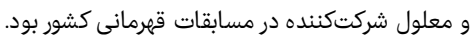

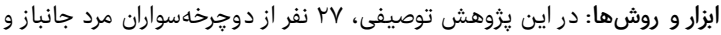

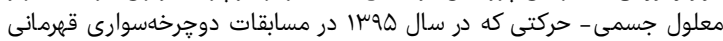

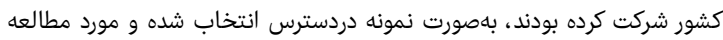

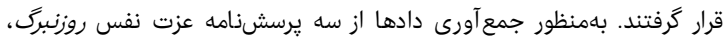

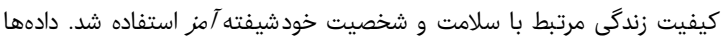

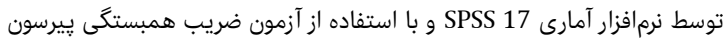

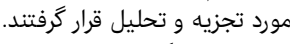

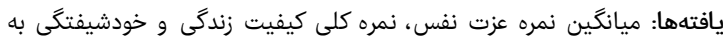

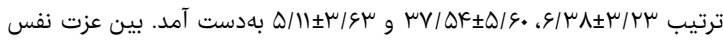

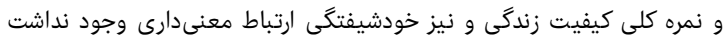

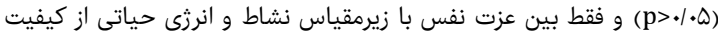

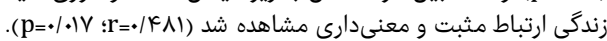

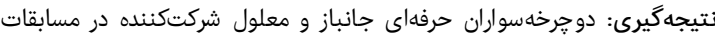

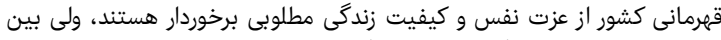

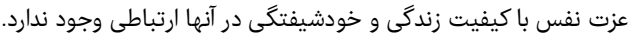

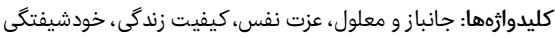

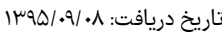

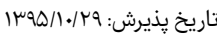

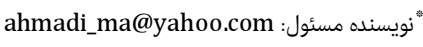

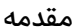

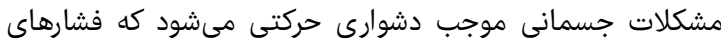

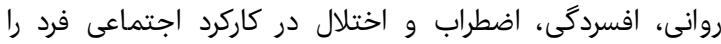

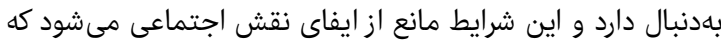

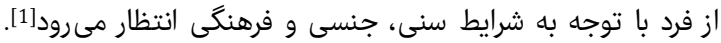

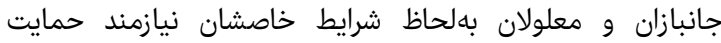

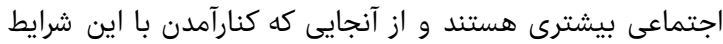

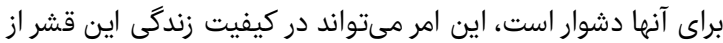

جامعه اثركذار باشد [2].

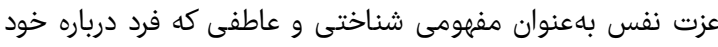

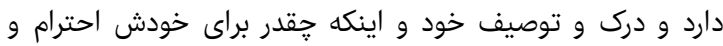

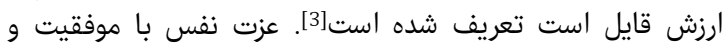

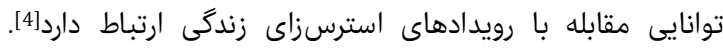

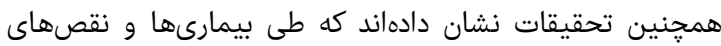

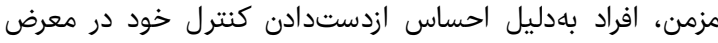

فصل نامه علمى - يزووهشى طب جانباز 


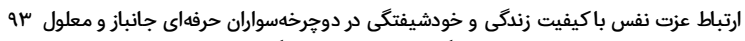

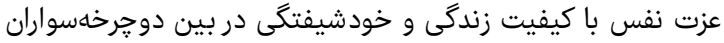
جانباز و معلول استفاده شد.

يافتهها

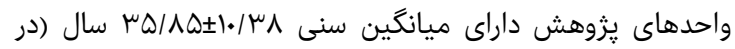

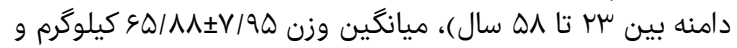

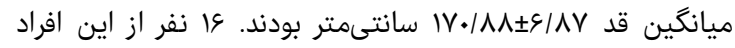

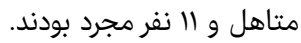

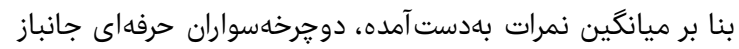

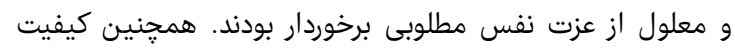
زندگى آنها در سطح خوبى قرار داشت و فاقد شخد شخصيت خودشيفته

بودند.

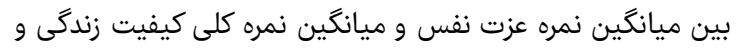

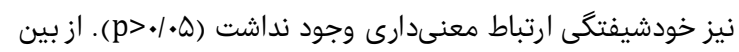

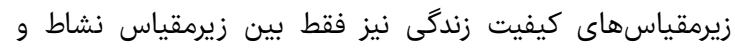

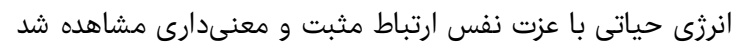

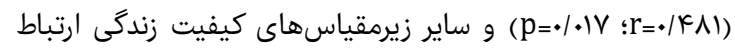

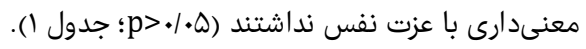

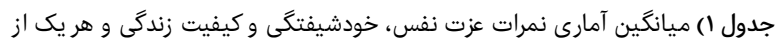

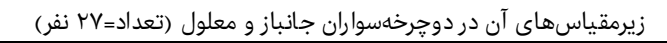

\begin{tabular}{|c|c|}
\hline ميانكين نمرات & شاخصها \\
\hline$s / \mu^{\mu} \wedge \pm \mu / \kappa \mu$ & عزت نفس \\
\hline \multirow[t]{2}{*}{$\Delta / \| \pm \mu / \varepsilon \mu$} & خودشيفتگى \\
\hline & كيفيت زندگىى \\
\hline r/GY & مولفه درك كلى از سلامت خود \\
\hline$F / \Delta K \pm I / \mu \Delta$ & مولفه عملكرد فيزيكى \\
\hline$\mu / \Delta \cdot \pm \cdot / \Lambda \Lambda$ & مولفه سلامت جسمانى \\
\hline$\Psi / \Delta K_{ \pm} \cdot / V V$ & مولفه مشكلات هيجانى \\
\hline$\kappa / r q \pm \cdot / \wedge \Delta$ & مولفه درد جسمانى \\
\hline$k / V \cdot \pm 1 / \cdot k$ & مولفه عملكرد اجتماعى \\
\hline$\mu / q \Delta \pm . / १ \Delta$ & مولفه نشاط و انرزى حياتى \\
\hline$q / r \Delta \pm 1 / R \Delta$ & مولفه سلامت روان \\
\hline$r y / r \Delta \pm \mu / 19$ & بعد سلامت روانى كل \\
\hline $1 Q / 9 \Delta \pm r / V V$ & بعد سلامت جسمانى كل \\
\hline$\mu V / \Delta K \pm \Delta / \varphi$. & نمره كلى \\
\hline
\end{tabular}

نتايج مطالعه حاضر نشان داد كه دوجرخهسواران حرفهاى جانباز و

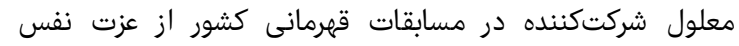

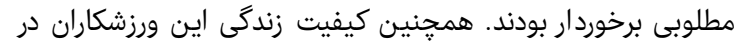

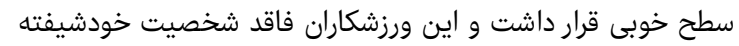

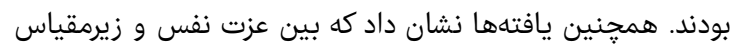

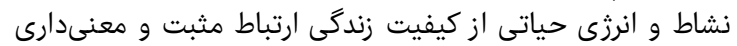

وجود دارد.

نوسك و همكاران نشان دادند كه زنان با معلوليت جسمى - حركتى

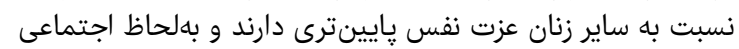

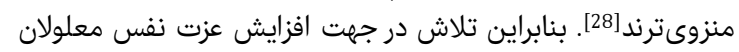

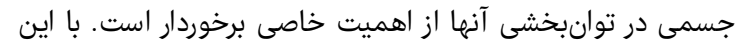

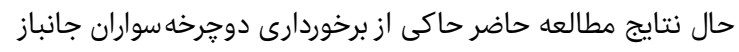

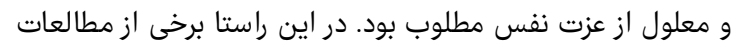

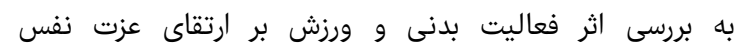

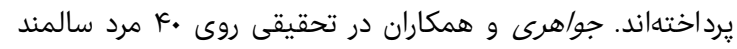

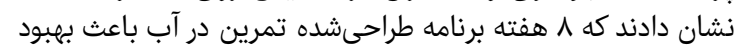

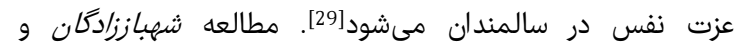

آكاهانه براى شركت در مطالعه، نداشتن بيمارى روانشناختى،

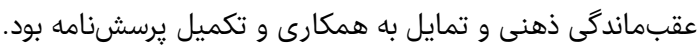

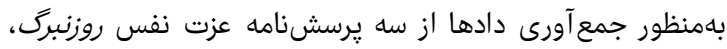

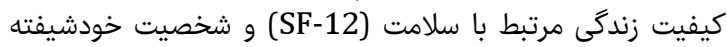

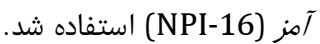

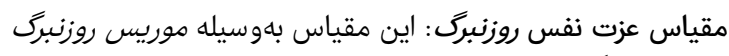

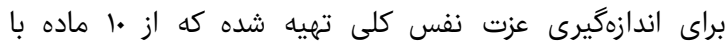

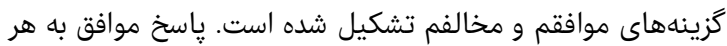

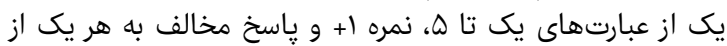

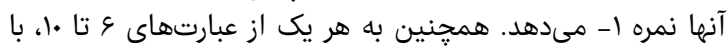

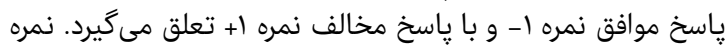

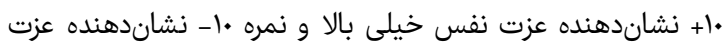

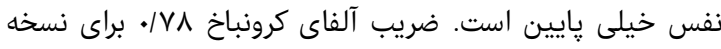

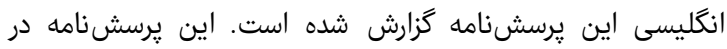

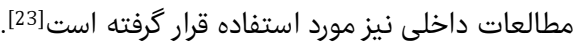

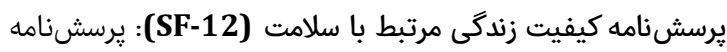

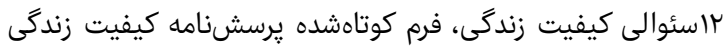

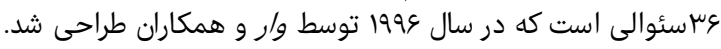

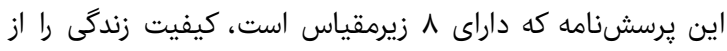

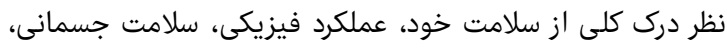

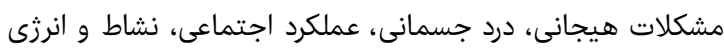

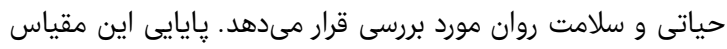

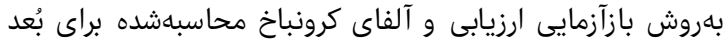

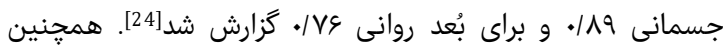

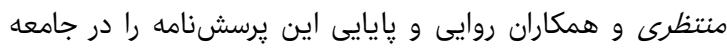

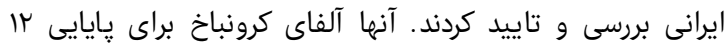

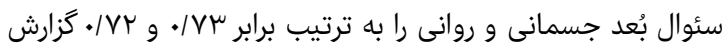

نمودند[25].

يرسشنامه شخصيت خودشيفته آمز (NPI-16): از پرسشنال

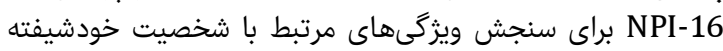

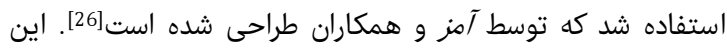

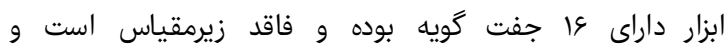

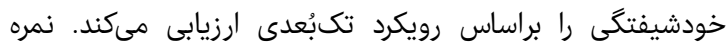

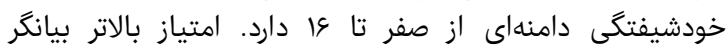

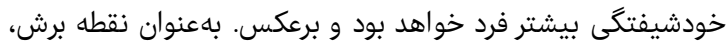

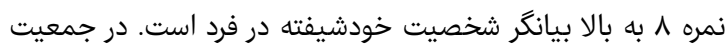

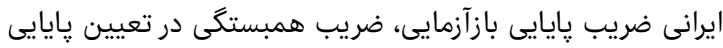

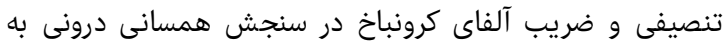

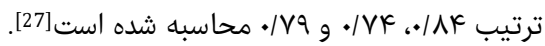

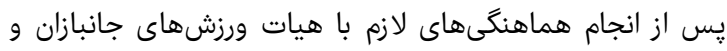

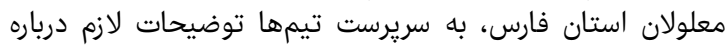

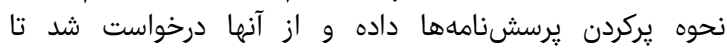

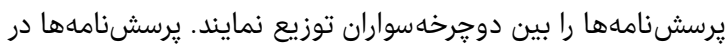

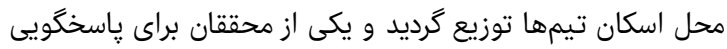

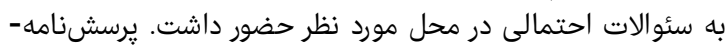

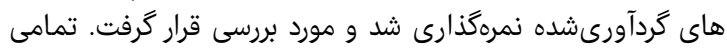

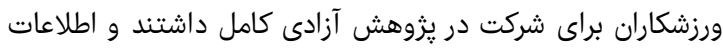

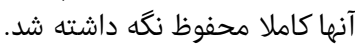

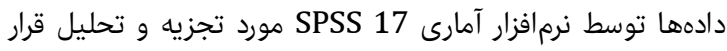

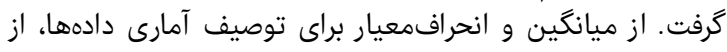

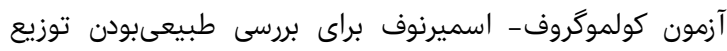

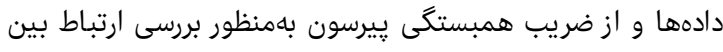

Volume 9, Issue 2, Spring 2017 
يافتههاى اوليه نشاندهنده يك ارتباط قوى بين خودشيفتگى و

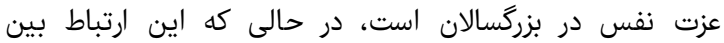

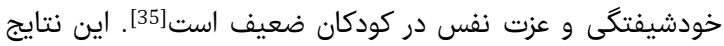

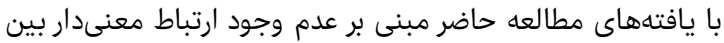

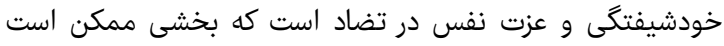

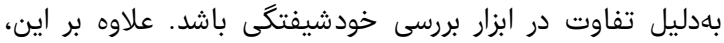

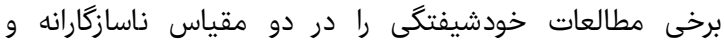

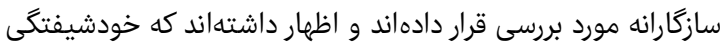

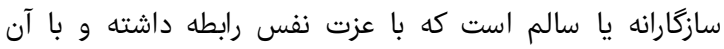

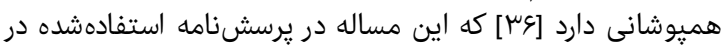

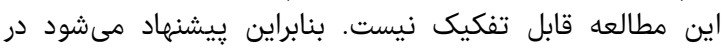

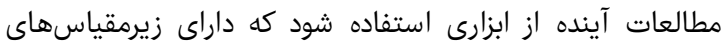

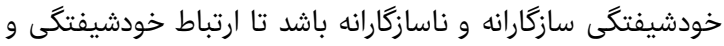

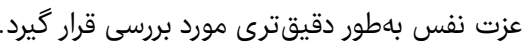

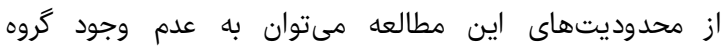

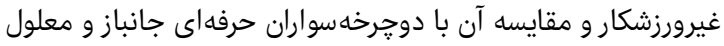

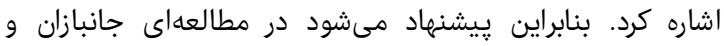

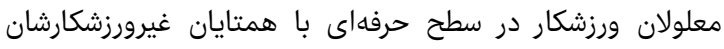
مقايسه شوند تا اثر ورزش بلهور در سبارزترى بررسى شود.

نتيجه دوجرخهسواران حرفهاى جانباز و معلول شركتئنئنده در مسابقات

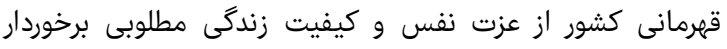

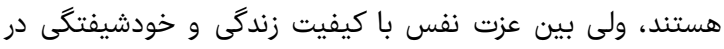

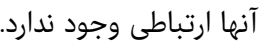

تشكر و قدردانى: نويسندگان مقاله بر خود لازم مىدانند از تمامى

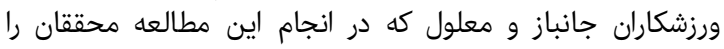

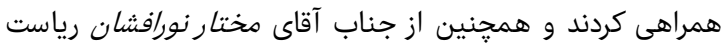

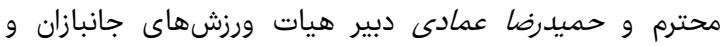
معلولان فارس كه با محققان همكارى لازم را داشتند كمال سياس ورناس و قدردانى را بهعمل آورند. تاييديه اخلاقى: مطالعه حاضر داراى تاييديه آلهايه اخلاقى با كد

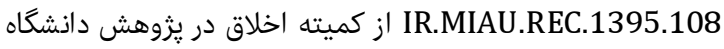

آزاد واحد مرودشت است.

تعارض منافع: هيج گَونه تعارض منافع توسط نوشيسندگان بيان نشده است.

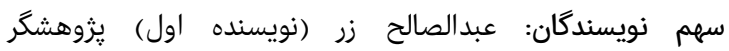

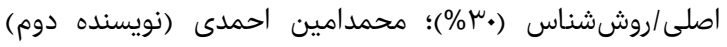

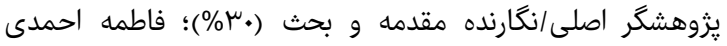

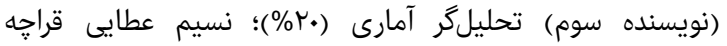

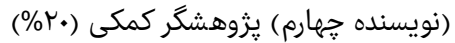

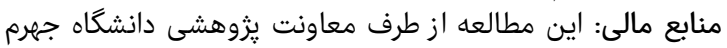

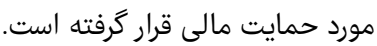

1- Hakim Javadi M, Lavasani M, Haghighatgoo M, Zabardast O. Relationship among depression, anxiety, stress and personality in veteran children. Iran J War Public Health. 2010;3(1):9-16. [Persian]

2- Nabatian E, Ghamarani A, Zakerian M, Mahdizadeh I. Relationship between Spiritual health With quality of life veterans and disabled Birjand. Iran J of War and Public Health. 2013;5(2):35-9. [Persian]

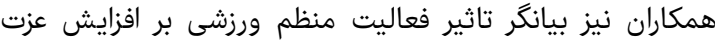

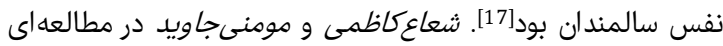

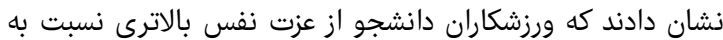

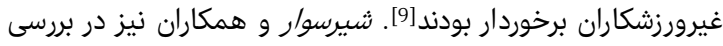

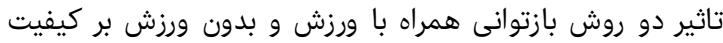

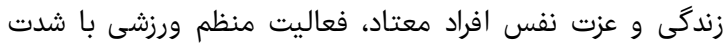

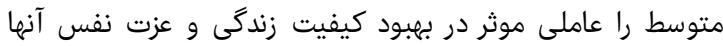

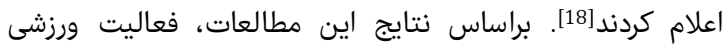

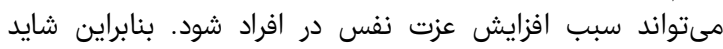

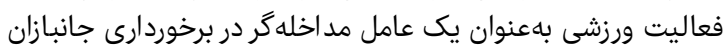

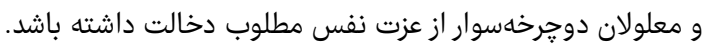

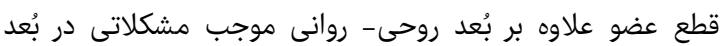

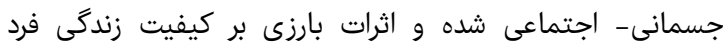

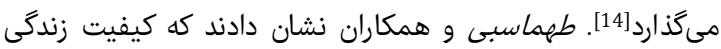

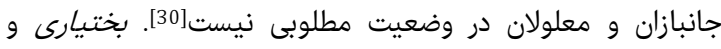

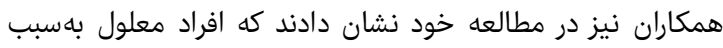

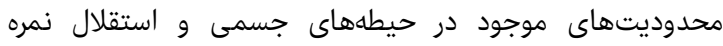

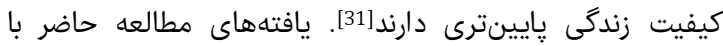

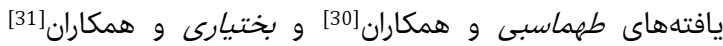

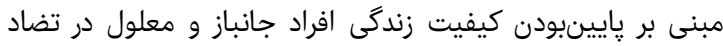

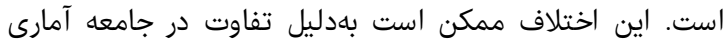

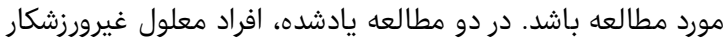

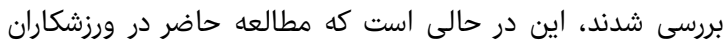

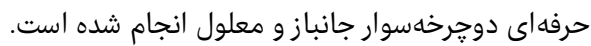

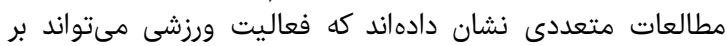

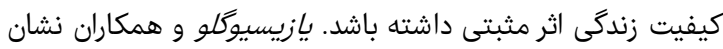

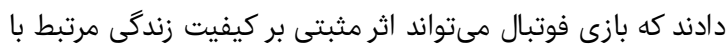

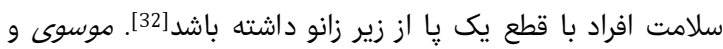

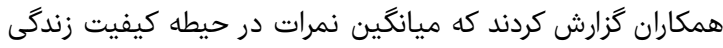

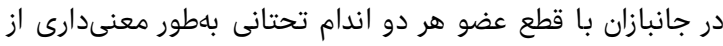

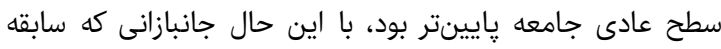

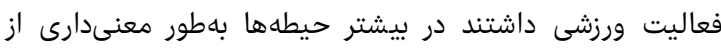

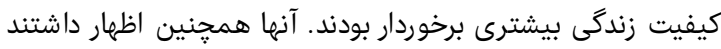

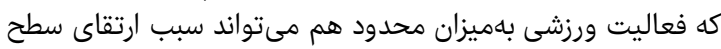

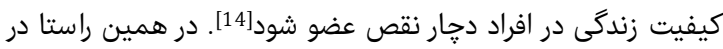

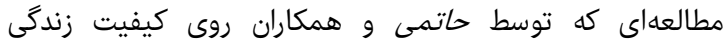

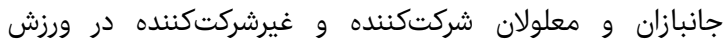

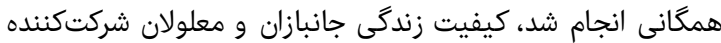

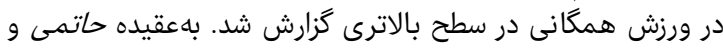

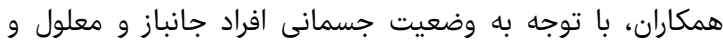

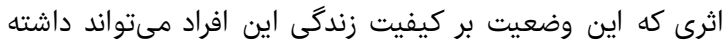

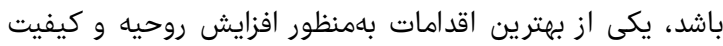

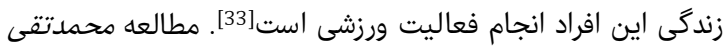

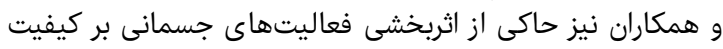

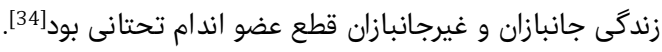

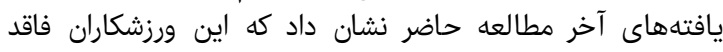

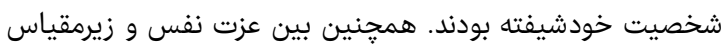

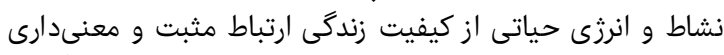

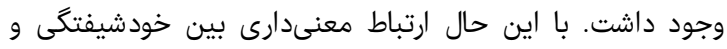

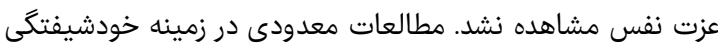

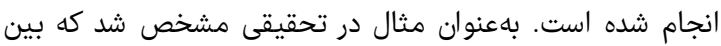
خودشيفتگى و عزت نفس رابطه مثبت معنى اسدارى وجود دارد[22]. 


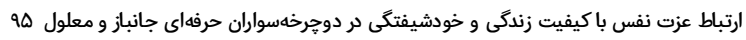
19- Hasanvand NZ, Javanmard K, Goodarzi M. Validation of the narcissistic personality inventory-40. J Psychol. 2015;19(1):102-18. [Persian]

20- Young SM, Pinsky D. Narcissism and celebrity. J Res Personal. 2006;40(5):463-71.

21- Raskin R, Novacek J, Hogan R. Narcissism, selfesteem, and defensive self-enhancement. J Pers. 1991;59(1):19-38.

22- Payvastegar M, Yazdi M, Mokhtari L. The relationship of narcissism, self esteem and aggression among adolescence girls and their comparisons in early, middle and late adolescence. Psychol Stud. 2012;7(1):130-47. [Persian]

23- Tavan B, Jahani F, Rafeei M. The Relationship between self-esteem and happiness among students of Arak University of Medical Sciences. Iran J Med Educ. 2014;14(6):474-82. [Persian]

24- Ware J Jr, Kosinski M, Keller SD. A 12-Item shortform health survey: Construction of scales and preliminary tests of reliability and validity. Med Care. 1996;34(3):220-33.

25- Montazeri A, Vahdaninia M, Mousavi SJ, Omidvari S. The Iranian version of 12 -item Short Form Health Survey (SF-12): Factor structure, internal consistency and construct validity. BMC Public Health. 2009;9:341.

26- Ames DR, Rose P, Anderson CP. The NPI-16 as a short measure of narcissism.J Res Personal.2006;40(4):440-50. 27- Mohammadzadeh A. Iranian validation of the narcissistic personality inventory-16. J Fundam Ment Health. 2010;11(4):274-81. [Persian]

28- Nosek MA, Hughes RB, Swedlund N, Taylor HB, Swank P. Self-esteem and women with disabilities. Soc Sci Med. 2003;56(8):1737-47.

29- Hashemi Javaheri AA, Mohamad Rahimi N, Ebrahimi Atri A. The effect of physical activity in the water on selfesteem. Iran J War and Public Health. 2012;4(1):32-9. [Persian]

30- Tahmasebi H, Abasi E, Zafari M, Darvish Gkezri H. Comparison of the quality of life of paraplegic veterans and disables; case study of mazandaran province, Iran. Iran J War Public Health. 2016;8(3):171-6. [Persian] 31- Bakhtiyari M, Salehi M, Zayeri F, Mobasheri F, Yavari P, Delpishe A, et al. Quality of life among disabled and healthy individuals a comparative study. Iran J Epidemiol. 2012;8(2):65-72. [Persian]

32- Yazicioglu K, Taskaynatan MA, Guzelkucuk U, Tugcu I. Effect of playing football (soccer) on balance, strength, and quality of life in unilateral below-knee amputees. Am J Phys Med Rehabil. 2007;86(10):800-5.

33- Hatami S, Saboonchi R, Sekhavat A, Ahar M, Musavi S. Comparing the quality of life of the disabled participat and non participat in Popular Sports. J Res Life Sci Sport. 2012;2(7):29-36. [Persian]

34- Mohamadtaghi B, Shamsipour Dehkordi P, Hejazi Dinan P. Effectiveness of physical activity on quality of life and pain self-efficacy in veterans and non-veterans with amputations of lower limbs. Iran J War Public Health. 2016;8(2):95-103. [Persian]

35- Barry CT, Frick PJ, Killian AL. The relation of narcissism and self-esteem to conduct problems in children: A preliminary investigation. J Clin Child Adolesc Psychol. 2003;32(1):139-52.

36- Trumpeter NN, Watson PJ, O'Leary BJ, Weathington BL. Self-functioning and perceived parenting: Relations of parental empathy and love inconsistency with narcissism, depression, and self-esteem. J Genet Psychol. 2008;169(1):51-71.
3- Suonpää E, Kresanov K, Ouvinen-Birgerstam P, Piha J. Self-concept of Finnish children on the second grade of normal elementary school. Acta Paedopsychiatr. 1989;52(3):157-64.

4- Orth U, Luciano EC. Self-esteem, narcissism, and stressful life events: Testing for selection and socialization. J Pers Soc Psychol. 2015;109(4):707-21.

5- Samadi N, Safavi M, Mahmoodi M. The relationship between quality of life and self-esteem in patients with type 2 diabetes in Ardabil 2011: A short report. J Rafsanjan Univ Med Sci. 2013;12(3):251-6. [Persian] 6- Soheylizad M, Yahaghi Amjad E, Gholamaliee B. Relationship between self-esteem, resilience and quality of life in patients with type 2 diabetes in Hamadan in 2015. Pajouhan Sci J. 2016;15(1):1-8. [Persian]

7- Chen YS, Chen MC, Chou FH, Sun FC, Chen PC, Tsai KY, et al. The relationship between quality of life and posttraumatic stress disorder or major depression for firefighters in Kaohsiung, Taiwan. Qual Life Res. 2007;16(8):1289-97.

8- Hosseini M, Dejkam M, Mirlashari J. Correlation between academic achievement and self-esteem in rehabilitation students in Tehran University of Social Welfare \& Rehabilitation. Iran J Med Educ. 2007;7(1):137-42. [Persian]

9- Shoaa Kazemi M, Javid Momeni M. Comparative study of the relationship between brave behavior, self-esteem and religious orientation athletic and non-athletic students. Res Islamic Educ Issue. 2011;19(12):169-90. [Persian]

10- Pucci GC, Rech CR, Fermino RC, Reis RS. Association between physical activity and quality of life in adults. Rev Saude Publica. 2012;46(1):166-79.

11- Spirduso WW, Cronin DL. Exercise dose-response effects on quality of life and independent living in older adults. Med Sci Sports Exerc. 2001;33(6 Suppl):S598608.

12- Bakhshayesh H, Bahmani F, Kamali M. Comparative of Mental Health Disabled People Of Athletic And NonAthletic. Iran J War Public Health. 2012;5(1):22-6. [Persian]

13- Shojaei H, Sokhangoei Y, Soroush M, Forouzan A, Modirian E, Nejati V. Evaluation of sport injury incidence in veterans and disabled athletes during MILAD-eKOWSAR festival in Tehran. Iran J War Public Health. 2009;1 (3):25-36. [Persian]

14- Musavi B, Salimi F, Sorush M, Masumi M, Montezeri M. Quality of life in Iranian veterans with amputations in both lower limbs. Payesh. 2009;8(3):303-10. [Persian] 15- Mandani B, Hosseini SA, Saadat Abadi M, Farahbod M. Effect of group exercise program on quality of life in post-traumatic stress disorder war veterans. Iran J War Public Health. 2015;7(2):91-8. [Persian]

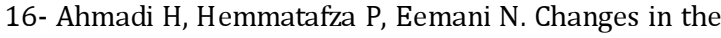
quality of life of veterans through regular physical activity. The First International Conference on the Role of the Islamic Revolution in Geometry Global Military Power. Tehran: Civilica; 2015.

17- Shahbazzadegan B, Farmanbar R, Ghanbari A, Atrkar Roshan Z, Adib M. The effect of regular exercise on selfesteem in elderly residents in nursing homes. J Ardabil Univ Med Sci. 2008;8(4):387-93. [Persian]

18- Shirsavar M, Amirtash A, Jalali S, Koushan M, Keavanlou F, Seyedahmadi M. Comparing the effectiveness of rehabilitation methods with and without exercise on quality of life and self-steam addicts. Q J Sabzevar Univ Med Sci. 2013;20(3):292-301. [Persian] 